\title{
A Decision Making: Implant or Endodontic Treatment? A Case Report
}

\section{Rodrigo Rodrigues Amaral ${ }^{1 *}$, Tiago Braga ${ }^{1}$, Stephen Cohen ${ }^{2}$, Ana Carolina Tocafundo Thompson ${ }^{3}$, Victor Couto Figueiredo ${ }^{3}$, Douglas Ferreira Freitas ${ }^{3}$ and Maria Ilma de Souza Côrtes ${ }^{3}$}

${ }^{1}$ School of Medicine and Dentistry Griffith University, Gold Coast, Queensland, Australia

${ }^{2}$ Department of Endodontics, Arthur Dugoni School of Dentistry, University of the Pacific, San Francisco, CA, USA

${ }^{3}$ Department of Dentistry, Pontifícia Universidade Católica de Minas Gerais, Belo Horizonte, Minas Gerais, Brazil

*Corresponding Author: Rodrigo Rodrigues Amaral, School of Medicine and Dentistry Griffith University, Gold Coast, Queensland, Australia.
Received: October 07, 2021

Published: October 23, 2021

C All rights are reserved by Rodrigo

Rodrigues Amaral., et al.

\begin{abstract}
The present report describes the successful treatment of a tooth with an endo-perio lesion and an extensive area of bone resorption. The possibility of extraction and dental implant was discussed with the patient. Chemomechanical preparation was performed, and canals were filled with bioceramic cement and gutta-percha cones. After 12 months, clinical and radiographic evaluations showed remarkable remineralization. The decision to perform the endodontic treatment or implant was based on scientific evidence and correct clinical assessment.
\end{abstract}

Keywords: Calcium Silicate; Dental Restoration; Endodontics; Periodontics; Photochemotherapy

\section{Introduction}

The success of endodontic treatment is directly related to cleaning, shaping, and sealing of the root canal system, which when performed correctly, leads to elimination of microorganisms and their toxins. However, success may be hampered because of anatomical complexity, strong root curvature, ramifications, and location of the apical foramen [1,2].

Over the last decade, there has been a paradigm shift with dental implant replacement being considered equal or even superior to the maintenance of natural teeth with a questionable prognosis. This has led to a dilemma among dentists regarding the correct clinical judgment and acceptable treatment [3].

The diagnosis of the endo-perio lesion applies to lesions originating from the inflammatory products found simultaneously in the pulp and surrounding periodontal tissues. The relationship between pulp and periodontal tissues was first described by Simring and Goldberg in 1964. A detailed comparative analysis is required of the indications, contraindications, risks and benefits of tooth main- 
tenance or an implant replacement. Of course, the patient must be informed of both treatment options in lay terms [4-6].

Advances in materials and technology in endodontics have increased the success rate of predictable outcomes and decreased the treatment time. In vivo research studies have reported that endodontic treatment procedures with adjunctive antimicrobial photodynamic therapy (aPDT) were successful $[7,8]$.

Recently, bioceramic materials have been introduced in endodontic therapy [9]. These materials contain bioactive and ceramic components with remarkable characteristics, e.g. ease of handling, dimensional stability, good flow and sealing activity, alkaline $\mathrm{pH}$ (which makes the environment inhospitable to antimicrobial proliferation), biocompatibility, and bioactivity $[9,10]$.

The treated root canal could be re-contaminated if the tooth is not promptly restored ${ }^{11}$ or if a temporary restorative material fails to promote strong marginal sealing. Hence, a permanent restoration is essential for long-term success [11].

\section{Case Report}

A 23-y.o woman was referred to have tooth \#19 evaluated. Her medical history was unremarkable and within normal limits. Clinical examination revealed an extensive amalgam restoration, dental mobility, and an 8-mm deep periodontal pocket on the buccal and lingual accompanied by sinus tracts. Radiographic examination revealed a thickened periodontal ligament (PDL) and an extensive radiolucent area around the root's furcation and periapical regions (Figure 1A). Tooth \#19 did not respond to the cold-thermal testing (Endo Frost, Germany); both percussion and palpation tests elicited positive responses. Under high magnification the clinical crown appeared to be completely intact and there was no radiographic evidence of root fracture. A diagnosis of an endo-perio lesion with extensive bone loss was made [12]. The possibility of extraction and implant replacement were discussed with the patient. After considering the risks, benefits and alternative the patient elected to try to save the tooth through endo-perio treatment.

Endodontic treatment was started after the patient signed an informed consent form (very important in today's litigious times). Local anesthesia (2\% lignocaine with epinephrine 1:100,000) of the inferior alveolar nerve and isolation with a rubber dam were achieved before treatment was initiation. The amalgam restoration was removed to achieve proper access to the root canals. Canal irrigation was conducted with a $5.25 \%$ sodium hypochlorite $(\mathrm{NaOCl})$ alternating with 17\% ethylenediaminetetraacetic acid (EDTA). 5.25\%. Stainless steel K-files \#10 and \#15 (Dentsply Maillefer, Ballaigues, Switzerland) were used to establish the glide path and reach the working length (WL). All root canals were instrumented sequentially with the files extending up to the WL. The mesial and distal roots were shaped using the rotary instrumentation technique with ProGlider and Pro Taper Next (Dentsply Maillefer, Ballaigues, Switzerland) as per the manufacturer's recommendations. Irrigation with $3 \mathrm{~mL}$ of $5.25 \% \mathrm{NaOCl}$ solution was performed after each instrument using a 30-gauge needle (NaviTip ${ }^{\circledR}$, Ultradent, USA) attached to a sterile disposable plastic syringe [13]. After completion of cleaning and shaping, the root canals were irrigated with $3 \mathrm{~mL}$ of 17\% EDTA solution ( $\mathrm{pH} 7.4$ ) for three minutes. This was followed by final irrigation with $3 \mathrm{~mL}$ of $5.25 \% \mathrm{NaOCl}$ solution and passive ultrasonic irrigation with an insert E1-Irrisonic ultrasonic tip (Helse Ultrasonic, Santa Rosa de Viterbo, Brazil) with 10\% of power for 3 cycles of 20 seconds. A $3 \mathrm{~mL}$ solution of $3 \%$ hydrogen peroxide was placed inside the root canal and left for 1 minute to eliminate any residual $\mathrm{NaOCl}$ solution. The root canals were irrigated with $0.005 \%$ methylene blue solution (Chimiolux, DMC, São Carlos, Brazil) used as a photosensitizer (PS). The Twin Flex laser equipment (MMOptics, São Carlos, Brazil) at a 660-nm wavelength and $100-\mathrm{mW}$ power was used for 90 seconds at $9.0 \mathrm{~J}$ of energy. A 300- $\mu$ m light diffuser (MMOptics, São Carlos, Brazil) was inserted into the root canal, $2 \mathrm{~mm}$ short of the WL. There was a 2-min wait between the application of the PS and laser activation. After laser irradiation, the root canals were irrigated with $3 \mathrm{~mL}$ of $\mathrm{NaOCl}$ solution to remove any residual methylene blue solution, and the canals were dried with FM paper points (Dentsply Latin America, Petrópolis, RJ, Brazil). The canals were then filled with calcium hydroxide paste UltraCal XS (Ultradent, South Jordan, UT, USA), and the access opening was sealed with a temporary restorative material (IRM; Dentsply Latin America, Petrópolis, RJ, Brazil) (Figure 1B). The intracanal medication was kept for 90 days without any change because patient could not return earlier. In the second visit, aPDT was performed following the same procedures as previously described. A remarkable bone repair was noted on the periapical radiograph of the gutta-percha trial (Figure 1C). Each root canal was sealed with FM gutta-percha cones $1 \mathrm{~mm}$ short of the WL by 
applying warm vertical compaction and bioceramic cement (BioCSealer Angellus, Londrina, Panará, Brazil) (Figure 1D). The patient was immediately referred to have the crown restored promptly to avoid any crown fracture or re-contamination of the root canals. The proposed treatment plan was to create a ceramic onlay. Gutta percha was removed using heat conductors and drills; $5 \mathrm{~mm}$ was left at the end of the canal. A dental pin, Exacto \# 1 (Angellus, Londrina, Brazil), was selected (Figure 2A), and conditioning with the substrate with 35\% phosphoric acid followed by self-adhesive resin cement RelyX ${ }^{\mathrm{TM}}$ U200 (3M ESPE, USA) and photoactivated for 40 seconds with a $1200 \mathrm{~mW} / \mathrm{cm}^{2}$ light-emitting diode device (Radii-Cal, SDI, Australia). The coronal part was reconstructed with composite resin Filtek Z-350 XT A3B (3M ESPE, St Paul, MN, USA). The amalgam restoration was removed and the onlay preparation was completed with the diamond drills \# 4138 and \# 3131 (KG Sorensen) (Figure 2B). The impression was taken with the aid of retracting threads, and the plaster model obtained was scanned for three-dimensional virtual planning (Figure 2C-2E). The images were processed in ExoCad software, and the generated file was milled in an IPS e.max CAD MT block and then sintered in the oven (programat IP 3010). The permanent restoration was cemented using Variolink N color A3 dual cement (Figure 2F), followed by the final photoactivation for 40 seconds on each face of the prosthesis as per the manufacturer's guidelines. After cementation, the rubber dam was removed, and an occlusal adjustment was made. After 12 months, the patient returned for clinical and radiographic evaluation (Figure 3). The periapical radiograph showed apical and furcation bone repair. The tooth was asymptomatic; there was no pain with vertical/horizontal percussion or palpation. Periodontal probing was within normal limits (WNL), mobility was WNL; the sinus tract closed and there was complete remineralization clearly indicating the success of the endodontic and restorative treatment.

\section{Discussion}

The diagnosis of a primary endodontic lesion with secondary periodontal involvement should only be made after gathering a thorough patient history along with comprehensive clinical testing and radiographic assessment. This disease entity is clinically and radiographically very similar to a primary periodontal disease [14]. Sometimes, dentists are faced with the difficult choice of performing endodontic treatment to preserve a tooth with a questionable prognosis or replacing it with a dental implant [15].

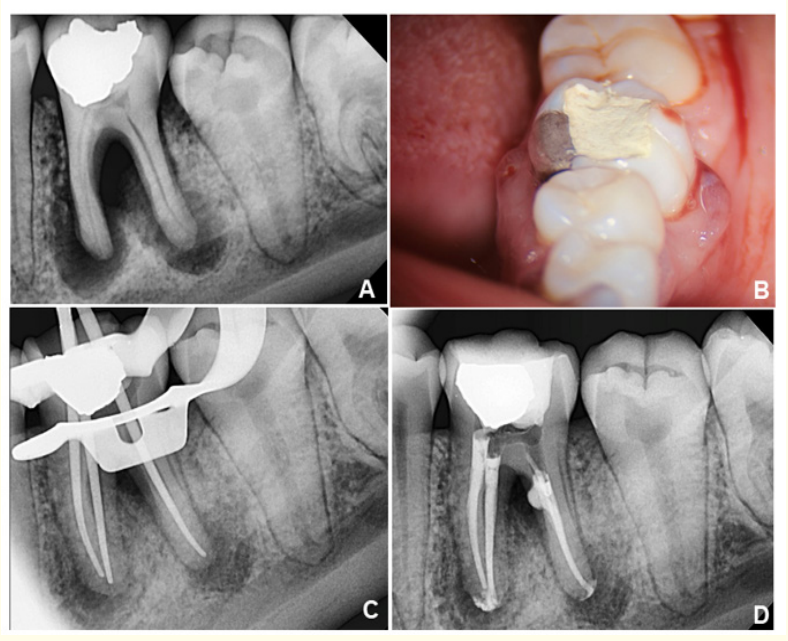

Figure 1: Sequence of endodontic treatment. A- Initial radiograph of tooth 19 note the extensive radiolucent area around the root's furcation and periapical region); B- Tooth 19 after first visit (Note the presence of fistulas on the buccal and lingual surfaces); C- Gutta-percha trial radiograph (Note the remarkable repair in the periapical and furcation areas); D- Final radiograph.
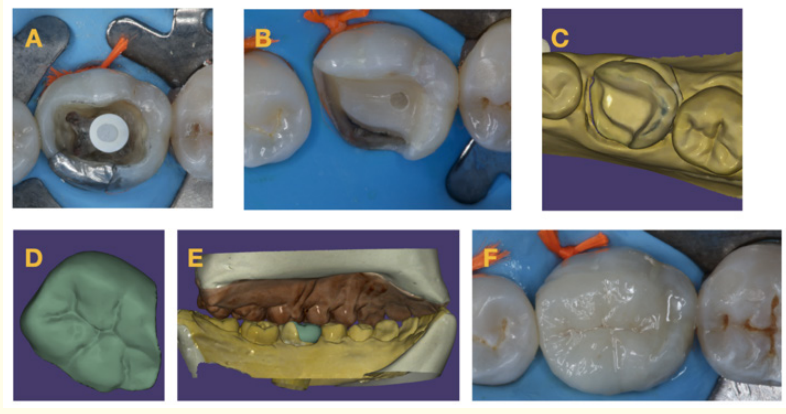

Figure 2: Restorative treatment steps. A- Intra-root retainer adapted in the distal canal; B- Onlay preparation completed; C- Occlusal view of the virtual model; D- Restoration ready for milling; E - Lateral (lingual) view in occlusion with the defined restoration; F- Occlusal view after cementation. 


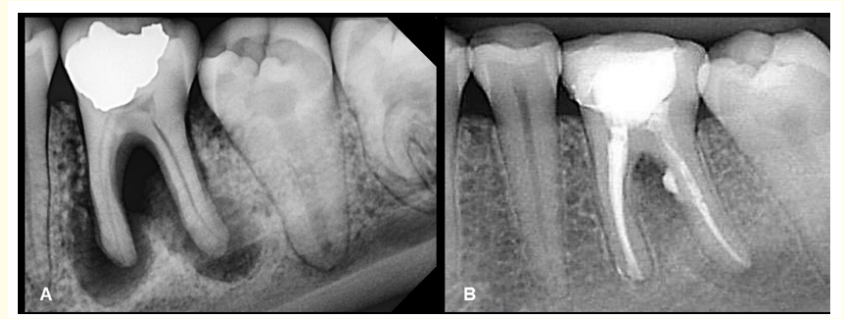

Figure 3: Periapical x-rays comparing pre- and post-op results. A-Pre-operative B- Post-operative, after 12 months of follow-up, noting remarkable repair in the periapical and furcation areas.

Despite the evolution of implant dentistry as a promising method for replacing missing teeth, the associated complications and failure rates are still problems that every implantologist has to face in clinical practice [16].

In this case, the patient's consent and the clinical diagnosis were of crucial importance in the decision- making process. The implant option was not accepted by the patient. Despite critical clinical conditions including severe mobility Class 3 , buccal and lingual sinus tracts, and deep periodontal pockets on two sides of the tooth, extensive bone loss was not present around other teeth.

The use of calcium hydroxide as an inter-appointment medication was important to maximize bacterial reduction due to the extent of tooth contamination and draining sinus tracts. Patient's obligations or geographical location may make it almost impossible to refresh the calcium hydroxide medication in the root canals [17]. During the interval between visits and after the endodontic treatment was concluded, there was no need for periodontal therapy. The case was not diagnosed as a primary advanced periodontal disease. The prognosis of primary endodontic disease with secondary periodontal involvement depends on the periodontal condition [18].

The aPDT has emerged as a promising adjunct to root canal treatment. It involves the use of a photosensitizer that is activated by light at a specific wavelength in the presence of oxygen. Energy transference from the activated oxygen results in the formation of oxygen species such as singlet oxygen and free radicals, highly re- active and harmful to proteins, lipids, nucleic acids and other microbial cellular components [18]. According to Garcez., et al. 2008, aPDT added to endodontic treatment leads to a significant decrease in bacterial load, and this could be an appropriate approach for the treatment of oral infections [7]. A systematic review investigated the effect of aPDT on bacterial load reduction, and all included studies that showed a positive effect on the reduction of microbial load ranging from 91.3 to $100 \%$ [19].

Bioceramic cements materials have important characteristics, such as ease of handling, dimensional stability, good flow, sealing activity, alkaline pH (makes the environment inhospitable for antimicrobial proliferation), biocompatibility, and bioactivity [20,21]. Because of the hydrophilic nature of Bioceramic cements, they can be easily applied in the presence of water, blood, or other dentinal fluids [22]. Bio-C Sealer cement was used in this case because of its beneficial properties. According to Zordan-Bronzel., et al. 2019, the Bio-C Sealer offers faster setting time, adequate alkalization capacity, flow, radiopacity, and dimensional stability [23].

Another critical factor for the success of the endodontic treatment is the restoration of the tooth. Epidemiological studies [2426] have evaluated the importance of a proper crown restoration placed in a timely way following endodontic therapy and have concluded that it is vital for treatment success. Incorporation of automation procedures for dental restorations has enabled a great reduction in manufacturing time and greater control of variables in the production protocol. The laboratory cost has become an important factor in planning, and automation helps in efficient and economical production. Flaws and disadvantages have been practically eliminated with the CAD/CAM technology. Through this system, it is possible to fabricate restorations with precise adjustment and greater durability [27-30].

\section{Conclusion}

This report illustrates a case that initially appeared to be a clinical failure and how it became an endodontic and restorative success, with clinical and radiographic evidence of bone repair after 12 months. The decision to perform the endodontic treatment was based on scientific evidence and correct clinical assessment.

\section{Conflict of Interest Statement}

There are no conflicts of interest to declare. 


\section{Bibliography}

1. Peters OA. "Current challenges and concepts in the preparation of root canal systems: a review". Journal of Endodontics 30 (2004): 559-567.

2. Siqueira Jr JF and Rôças IN. "Clinical implications and microbiology of bacterial persistence after treatment procedures". Journal of Endodontics 34 (2008): 1291-1301.

3. John V., et al. "Implant or the natural tooth - A contemporary treatment planning dilemma?" Australian Dental Journal 52.1 (2007): 138-150.

4. Simring M and Goldberg M. "The pulpal pocket approach: retrograde periodontitis". Journal of Periodontology 35 (1964): 22-48.

5. Raja VS., et al. "The periodontal - endodontic continuum: A review". Journal of Conservative Dentistry 11 (2008): 54-62.

6. Iqbal MK and Kim S. "A review of factors influencing treatment planning decisions of single-tooth implants versus preserving natural teeth with nonsurgical endodontic therapy". Journal of Endodontics 34 (2008): 519-529.

7. Garcez AS., et al. "Antimicrobial effects of photodynamic therapy on patients with necrotic pulps and periapical lesion". Journal of Endodontics 34 (2008): 138-142.

8. Amaral RR., et al. "Antimicrobial Photodynamic Therapy associated with long term success in endodontic treatment with separated instruments: A case report". Photodiagnosis and Photodynamic Therapy 26 (2019): 15-18.

9. Al-Haddad A and Che Ab Aziz ZA. "Bioceramic-Based Root Canal Sealers: A Review". International Journal of Biomaterials (2016): 1-10

10. Candeiro GTM., et al. "Cytotoxicity, genotoxicity, and antibacterial effectiveness of a bioceramic endodontic sealer". International Endodontic Journal 49 (2015): 858-864.

11. Torabinejad M., et al. "In vitro bacterial penetration of coronally unsealed endodontically treated teeth". Journal of Endodontics 16 (1990): 566-569.
12. Steffens JP and Marcantonio RAC. "2018 Classification of Periodontal and Periimplantar Diseases and Conditions: a Practical Guide and Key Points". Journal - Revista de Odontologia da UNESP 47 (2018): 189-197.

13. Amaral RR., et al. "Quantitative assessment of the efficacy of two different single-file systems in reducing the bacterial load in oval-shaped canals: A clinical study". Journal of Endodontics 46 (2020): 1228-1234.

14. Rotstein I and Simon JHS. "Diagnosis, prognosis, and decision making in the treatment of combined periodontal-endodontic lesions". Periodontology 200034 (2004): 265-303.

15. Zitzmann NU., et al. "Strategic considerations in treatment planning: Deciding when to treat, extract or replace a questionable tooth". Journal of Prosthetic Dentistry 104 (2010): 8091.

16. Albrektsson $\mathrm{T}$ and Donos N. "Implant survival and complications. The Third EAO consensus conference 2012". Clinical Oral Implants Research 23 (2012): 63-65.

17. Vera J., et al. "One- versus Two-visit Endodontic Treatment of Teeth with Apical Periodontitis: A Histobacteriologic Study". Journal of Endodontics 38 (2012): 1040-1052.

18. Mhairi RW. "The pathogenesis and treatment of endo-perio lesions". CPD Dent 2 (2001): 91-95.

19. Chrepa V., et al. "The Effect of Photodynamic Therapy in Root Canal Disinfection: A Systematic Review". Journal of Endodontics 40 (2014): 891-898.

20. Koch KA., et al. "Bioceramic technology: closing the endo-restorative circle, Part I”. Dent Today 29 (2010): 100-105.

21. Koch KA., et al. "Bioceramic technology: closing the endo-restorative circle, part 2". Dent Today 98-100 (2010): 102-105.

22. Prati $\mathrm{C}$ and Gandolfi MG. "Calcium silicate bioactive cement: Biological perspectives and clinical applications". Dent Mater 31 (2015): 351-370.

23. Zordan-Bronzel CL., et al. "Cytocompatibility, bioactive potential and antimicrobial activity of an experimental calcium silicate-based endodontic sealer". International Endodontic Journal J 52 (2019): 979-986. 
24. Ray HA and Trope M. "Periapical status of endodontically treated teeth in relation to the technical quality of the root filling and the coronal restoration". International Endodontic Journal 28 (1995): 12-18.

25. Siqueira Jr JF., et al. "Periradicular status related to the quality of coronal restorations and root canal fillings in a Brazilian population". Oral Surgery, Oral Medicine, Oral Pathology, Oral Radiology, and Endodontology 100 (2005): 369-374.

26. Ng YL., et al. "Outcome of primary root canal treatment: systematic review of the literature - Part 2. Influence of clinical factors". International Endodontic Journal 41 (2008): 6-31.

27. Yara A., et al. "Correlation between accuracy of crowns fabricated using CAD/ CAM and elastic deformation of CAD/CAM Materials". Dental Materials Journal 23 (2004): 572-576.

28. Beuer F., et al. "Digital dentistry: an overview of recent developments for CAD/CAM generated restorations". British Dental Journal 204.9 (2008): 505-509.

29. Jain R., et al. "CAD-CAM the future of digital dentistry: a review". Annals of Prosthodontics and Restorative Dentistry 2.2 (2016): 33-36.

30. Baroudi K and Ibraheem SN. "Assessment of Chair-side Computer-Aided Design and Computer-Aided Manufacturing Restorations: A Review of the Literature". Journal of International Oral Health 7.4 (2015): 96-104.

Volume 5 Issue 11 November 2021

(C) All rights are reserved by Rodrigo Rodrigues Amaral., et al. 